\title{
Health Disparities Among Black Persons in the US and Addressing Racism in the Health Care System
}

\author{
Aaron E. Carroll, MD, MS
}

Department of Pediatrics, Indiana University School of Medicine, Indianapolis

The protests occurring across the US beginning the day after Memorial Day were sparked by a shocking video that captured how black persons are often grossly abused and even killed by a broken criminal justice system. In front of our eyes, George Floyd died on May 25, 2020, as a police officer pressed his knee on Floyd's neck as Floyd gasped, "I can't breathe."

In addition to attributing the cause of death to "cardiopulmonary arrest while being restrained by law enforcement officer(s)," Hennepin County prosecutors and an early account from the medical examiner in the case list other "significant conditions," including "arteriosclerotic and hypertensive heart disease."

At a time when many people in the US are awakening to the systemic racism in the criminal justice system, it is equally important to acknowledge its existence in the US health care system.

Research shows that because of systemic racism, black persons have higher levels of chronic illnesses compared with white persons. It is hard to fathom the gall it took to imply blame for Floyd's death on the very disparities in which racism plays so large a role.

Evidence has shown for decades that black persons are treated differently (worse) than white persons by the US health care system. In the notorious Tuskegee experiments, members of the US Public Health Service followed up black men infected with syphilis without treating them to observe how the disease took its course; the experiments ended in 1972, but their effects are still being felt. The US Agency for Healthcare Research and Quality has been tracking racial disparities since 2000. They still remain.

Every single time I hear a presentation or read a manuscript pointing out differences in how black persons are treated with respect to health care, I despair. It seems to me that anyone who does not know this yet is actively remaining ignorant and will not be convinced by 1 more study.

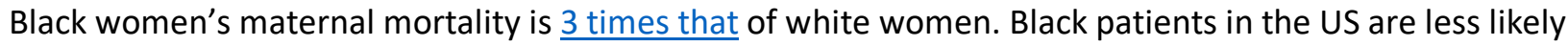
to receive proper care for diabetes, kidney disease, and various cancers even though they have higher rates of almost every disease.

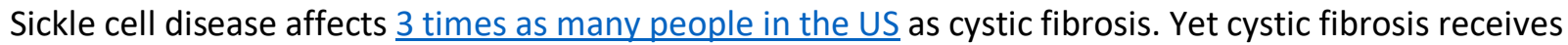
11 times as much funding per patient from the federal government, and 440 times more funding from foundations.

Black persons in the US face roadblocks in every aspect of health care and even in academic medicine. They are less likely to be able to access health care. If they are able to do so, they are less likely to get the care they need to remain healthy. They are less likely to succeed in the profession. They are less likely to be awarded grants. They are less likely to be promoted, and less likely to be in positions of leadership. There is evidence demonstrating all of this.

This is the author's version of the work published in final form as:

Carroll, A. E. (2020). Health Disparities Among Black Persons in the US and Addressing Racism in the Health Care System. JAMA Health Forum, 1(6), e200769-e200769.

https://doi.org/10.1001/jamahealthforum.2020.0769 
The coronavirus disease 2019 (COVID-19) pandemic has underscored the many levels of systemic racism. Black persons are more likely to have chronic conditions that lead to severe cases of COVID-19. They are more likely to hold lower-paying yet "essential" jobs that place them in harm's way, more likely to be reliant on public transportation where social distancing is hard, and more likely to live in housing that compounds all that risk.

Even before any protests began, black persons were dying of COVID-19 at rates twice the rate that one would expect based on their share of the US population. In Wisconsin, COVID-19 deaths among black persons comprise more than one-quarter of such deaths, even though they are only $6 \%$ of the population. In a study in Louisiana, more than $70 \%$ of deaths occurred in black persons despite their comprising only $30 \%$ of the studied population. Disproportionately black counties account for more than half of COVID-19 deaths nationwide, and wealth and access to health care do not seem to equalize things.

Should the protests following George Floyd's death cause transmission of infection, many will blame those who showed up. Those at higher risk-again, black persons-will be more likely to develop severe illness and die. This and all the other disparities are easily predicted, yet it seems, like so many US politicians, the health care community too often offers only "thoughts and prayers" rather than effecting change.

Part of public health is making sure the public is healthy. The US health care system has failed to do that with too many in this country. The US society in general and the health care community in particular need to acknowledge that so much of what is wrong with the health of black persons is the fault of the health care system and not of patients. "Personal responsibility" plays well, but it is often a way to blame the patient when the system fails to support their ability to care for themselves.

It is time to stop wasting time and money proving that disparities exist. It is clear that they do, and pointing out the problem is easy. It is time to do something about it, which is infinitely harder.

It is time to invest in public health to improve the ability of everyone to eat right and exercise regularly, and to quit smoking and drinking unsafely. Just telling them to do so is not enough. Massive investment into making it easier to do so is necessary.

It is time to make sure that everyone has access to the health care system and preventive care. The Affordable Care Act was necessary, but not sufficient. Too many still do not have access to Medicaid, and too many cannot afford care even when insured.

It is time to train physicians to avoid implicit and explicit racial bias when seeing patients. It is time to rebuild trust with black persons and for the health care community to own past mistakes and prevent them from happening again. And it is time to recognize that the reasons black persons fare so poorly with respect to health is because of disparities, not because they chose not to care for themselves, and to fix those disparities.

As efforts to contain the COVID-19 pandemic continue after protests subside, it is essential to recognize that systemic racism kills black persons through poor health as much as or even more than police brutality. It does so because society tolerates a system that sees them as expendable, even as it labels them essential. 
Article Information

Corresponding Author: Aaron E. Carroll, MD, MS, Indiana University School of Medicine, Department of Pediatrics, Ped-Comparative Effectiveness, HS 2000A PCER, Indianapolis, IN 46202 (aaecarro@iu.edu).

Conflict of Interest Disclosures: None reported. 